\title{
On- and off-line identification of linear state-space models
}

\section{MARC MOONEN $\dagger$, BART DE MOOR†, \\ LIEVEN VANDENBERGHE $\dagger$ and JOOS VANDEWALLE $\dagger$}

A geometrically inspired matrix algorithm is derived for the identification of statespace models for multivariable linear time-invariant systems using (possibly noisy)
input-output (I/O) measurements only. As opposed to other (mostly stochastic identification schemes, no variance-covariance information whatever is involved, and only a limited number of $\mathrm{I} / \mathrm{O}$-data are required for the determination of the system matrices. Hence, the algorithm can be best described and understood in the matrix formalism, and consists of the following two steps. First, a state vector sequence is realized as the intersection of the row spaces of two block Hankel matrices, constructed with $\mathrm{I} / \mathrm{O}$-data. Then the system matrices are obtained at once from the least-squares solution of a set of linear equations. When dealing with noisy data, this algorith draws its excellent performance from repeated use of the is easily applied to slowly time-varying systems using windowing or exponential weighting. These results are illustrated by examples, including the identification of an industrial plant.

1. Introduction

Identification aims at finding a mathematical model from the measurement record of inputs and outputs of a system. A state-space model is a most obvious choice for the mathematical representation because of its widespread use in system theory and control. However, reliable general purpose state-space identification schemes have not become standard tools so far, mostly due to the computational complexity involved (Ho and Kalman 1965, Kung 1978. Zeiger and McEwen 1974).

The theory of canonical correlation analysis, independently developed in the mid1930s by Hotelling (1936) and Obukhov (the idea of using singular value decomposition (SVD) to compute the principal angles and vectors being due to Bjorck and Golub (Golub and Van Loan 1983), has been intensively applied to the stochastic identification problem, where, as a major departure, canonical variate analysis is used to choose linear combinations of the past random process to optimally predict the future of the process. The analysis of a system in terms of past and future naturally leads to a state-space description (Akaike 1974, 1975, Baram 1981, Ramos and Verriest 1984, Larimore 1984). Nevertheless, the intensive use of covariance information is a major drawback in practice, since finite data records reveal only poor approximations for covariance matrices.

In this paper, a novel approach is presented, that resembles the canonical variate methods greatly, although no variance-covariance information is involved whatsoever, and only a finite number of input-output-(I/O)-data are required for the determination of the system matrices. The main step in the identification procedure consists of the SVD of a block Hankel matrix, constructed with I/O-data. As it turns

Received 12 April 1988

TESAT Katholieke Universiteit Leuven, K. Mercierlaan 94,3030 Heverlee, Belgium. 0020.71798953 .00 \& 1989 Taylor \& Francis Lid 
out that only the left singular basis is required, both the computational load and the noise sensitivity are considerably reduced. Moreover, the identification scheme is easily converted into an adaptive version. Section 2 briefly describes useful properties of dynamic systems that are used in $\S 3$ to show how a sequence of state vectors can be calculated. The system matrices are then identified by solving an overdetermined set of linear equations (§4). The off-line algorithm is summarized in $\S 5$, and converted nto an adaptive on-line algorithm for slowly time-varying systems in $\S 6$. Both strategies are illustrated by examples.

\section{Dynamic systems}

The most general linear discrete-time multivariable state-space model may be written as

$$
\begin{aligned}
x[k+1] & =A_{k} \cdot x[k]+B_{k} \cdot u[k]+w[k] \\
y[k] & =C_{k} \cdot x[k]+D_{k} \cdot u[k]+v[k]
\end{aligned}
$$

where $u[k], y[k]$ and $x[k]$ denote the input (m-vector), output (l-vector) and state vector at time $k$, the dimension of $x[k]$ being the minimal system order $n . A_{k}, B_{k}, C_{k}$ and $D_{k}$ are the unknown system matrices at time $k$ to be identified, only making use of recorded I/O-sequences $u[k], u[k+1], \ldots$ and $y[k], y[k+1], \ldots$ As it is obvious that only the observable part of the system can be identified from observed I/O-data, it is only the observable part of the system can be identified from observed I/O-data, it is
assumed that the system is completely observable, thus omitting the unobservable assumed that the system is completely observable, thus omitting the unobservable ccounting for measurement noise, process noise, model mismatch, etc. They are identified as the residuals of the set of equations that determine the system matrices (\$), and can thus be omitted for a while. Also, for the time being, we consider only time-invariant systems, so that the state-space equations eventually reduce to

$$
x[k+1]=A \cdot x[k]+B \cdot u[k]
$$

$$
y[k]=C \cdot x[k]+D \cdot u[k]
$$

We now state two important theorems that are used throughout.

\section{Theorem 1}

Sequences $u, y, x$ that satisfy (2), also satisfy the following general structured $\mathrm{I} / \mathrm{O}$ equation:

$$
Y_{h}=\Gamma_{i} \cdot X+H_{t} \cdot U_{h}
$$

$Y_{b}$ is a block Hankel matrix ( $i$ block rows, $j$ columns) containing the consecutive outputs ( $y[k]$ is an $l \times 1$ vector, where $l$ is the number of outputs)

$$
Y_{h}=\left[\begin{array}{cccc}
y[k] & y[k+1] & \ldots & y[k+j-1] \\
y[k+1] & y[k+2] & \ldots & y[k+j] \\
y[k+2] & y[k+3] & \ldots & y[k+j+1] \\
\vdots & \vdots & & \vdots \\
y[k+i-1] & y[k+i] & \ldots & y[k+j+i-2]
\end{array}\right]
$$

$U_{h}$ is a block Hankel matrix with the same block dimensions as $Y_{h}$, containing the consecutive inputs $(u[k]$ is an $m \times 1$ vector, where $m$ is the number of inputs)

$$
U_{h}=\left[\begin{array}{cccc}
u[k] & u[k+1] & \ldots & u[k+j-1] \\
u[k+1] & u[k+2] & \ldots & u[k+j] \\
u[k+2] & u[k+3] & \ldots & u[k+j+1] \\
\vdots & \vdots & & \vdots \\
u[k+i-1] & u[k+i] & \ldots & u[k+j+i-2]
\end{array}\right]
$$

$X$ contains consecutive state vectors

$$
X=[x[k] \quad x[k+1] \quad x[k+2] \quad \ldots \quad x[k+j-1]]
$$

$\Gamma_{1}$ is an extended observability matrix

$$
\Gamma_{i}=\left[\begin{array}{c}
C \\
C A \\
C A^{2} \\
\vdots \\
C A^{i-1}
\end{array}\right]
$$

Finally, $H_{1}$ is a lower triangular block Toeplitz matrix containing the Markov parameter

$$
H_{t}=\left[\begin{array}{ccccc}
D & 0 & 0 & \ldots & 0 \\
C B & D & 0 & \ldots & 0 \\
C A B & C B & D & \ldots & 0 \\
C A^{2} B & C A B & C B & \ldots & 0 \\
\vdots & \vdots & \vdots & & \vdots \\
C A^{i-2} B & C A^{i-3} B & C A^{i-4} B & \ldots & D
\end{array}\right]
$$

Proof

The proof is straightforward by repeated substitution of (2).

Instead of going into details, we loosely state that $i$ and $j$ should be chosen 'sufficiently large' (so that $Y_{h}$ and $U_{h}$ contain enough information on the system), and in particular $j \gg \max (m i, l i)$ ('very rectangular' block Hankel matrices), as this reduces both the computational load and the noise sensitivity (see below).

Theorem 2

Let $Y_{h}, U_{h}$ and $X$ be defined as in the previous theorem, and let $H$ denote the concatenation of $Y_{h}$ and $U_{h}$

$$
H=\left[\begin{array}{l}
Y_{h} \\
U_{h}
\end{array}\right]
$$


then, under the conditions that

(i) $\operatorname{rank}(X)=n$, i.e. all modes are sufficiently excited ( $n$ being the minimal system order), and

(ii) $\operatorname{span}_{\text {row }}(X) \cap \operatorname{span}_{\text {row }}\left(U_{h}\right)=\varnothing$, the following rank property holds

$$
\operatorname{rank}(H)=\operatorname{rank}\left(U_{h}\right)+n
$$

(iii) Also, when rank $\left(U_{h}\right)=m i=$ number of rows in $U_{h}$, this rank property reduces

$$
\operatorname{rank}(H)=m i+n
$$

Proof

From (3) it follows that

$$
Y_{h} \cdot U_{h}^{\frac{1}{h}}=\Gamma_{i} \cdot X \cdot U_{h}^{\frac{1}{h}}
$$

and then

$$
\operatorname{rank}\left(Y_{h} \cdot U_{h}^{1}\right)=\operatorname{rank}\left(\Gamma_{i} \cdot X \cdot U_{h}^{\frac{1}{h}}\right)
$$

where the columns of $U_{h}^{1}$ span the kernel of $U_{h}$ (not trivial since $\left.j \gg m i\right)$. Since $\Gamma_{i}$ has full column rank (cf. observability)

$$
\begin{aligned}
\operatorname{rank}\left(\Gamma_{i} \cdot X \cdot U_{h}^{1}\right) & =\operatorname{rank}\left(X \cdot U_{h}^{\frac{1}{h}}\right)-\operatorname{dim}\left[\operatorname{span}_{\text {col }}\left(X \cdot U_{h}^{\frac{1}{h}}\right) \cap\left(\operatorname{span}_{\text {row }}\left(\Gamma_{i}\right)\right)^{\perp}\right] \\
& =\operatorname{rank}\left(X \cdot U_{h}^{\frac{1}{h}}\right)-\operatorname{dim}\left(\operatorname{span}_{\text {col }}\left(X \cdot U_{h}^{1}\right) \cap \varnothing\right) \\
& =\operatorname{rank}\left(X \cdot U_{h}^{\frac{1}{h}}\right)
\end{aligned}
$$

By making use of condition (ii)

$$
\begin{aligned}
\operatorname{rank}\left(X \cdot U_{h}^{1}\right) & =\operatorname{rank}(X)-\operatorname{dim}\left[\operatorname{span}_{\text {row }}(X) \cap\left(\operatorname{span}_{\text {col }}\left(U_{h}^{1}\right)^{\perp}\right]\right. \\
& =\operatorname{rank}(X)-\operatorname{dim}\left[\operatorname{span}_{\text {row }}(X) \cap \operatorname{span}_{\text {row }}\left(U_{h}\right)\right] \\
& =\operatorname{rank}(X)
\end{aligned}
$$

Finally, under condition (i)

$$
\operatorname{rank}(X)=n
$$

By combining all the above equations, we obtain

$$
\operatorname{rank}\left(Y_{h} \cdot U_{h}^{1}\right)=n
$$

and this, in fact, means that the row space of $Y_{h}$ adds $n$ dimensions to the row space of $U_{h}$, which proves (4)

This theorem allows us to estimate the system order, prior to further identification of the system matrices.

Note on condition $(i)$ : $\operatorname{rank}(X)=n$, in other words all modes should be sufficiently excited (persistant excitation). When certain modes are not, i.e. unobservable in the I/O-data currently under investigation, they cannot be identified either and application of the above rank property will reveal too low a system order, this problem being inherent in system identification.
Note on condition (ii): When this condition is not satisfied, $\operatorname{rank}\left(X \cdot U_{h}^{1}\right)$ $<\operatorname{rank}(X)$ (rank cancellation), and again application of the rank property reveals an underestimation of the system order. However, it can be experimentally verified that rank cancellation is not generic, and the probability that rank cancellation occurs, decreases for fixed $i$ (number of rows in $U_{h}$ ) with increasing $j$ (number of columns in $U_{h}$ and $X$ ). (In a stochastic framework, this matter would be passed off easily by saying $E\left(x[k] \cdot u[k]^{\prime}\right)=0, E\left(x[k] \cdot u[k+1]^{\prime}\right)=0, \ldots$, where $E$ is the expectation operator.)

Note on condition (iii): Similar to the previous ones, this third condition is generically satisfied when the input is 'sufficiently exciting' (inherent in the identification problem).

In the following, it is always assumed that these three conditions are satisfied

\section{Determination of state vector sequence}

We now demonstrate how a sequence of state vectors may be calculated as the intersection of the row spaces of two block Hankel matrices, constructed from input-output vectors. Let $H_{1}$ and $H_{2}$ be the concatenation of $Y_{h 1}, U_{h 1}$ and $Y_{h 2}, U_{h 2}$ respectively

$$
H_{1}=\left[\begin{array}{c}
Y_{h 1} \\
U_{n 1}
\end{array}\right], \quad H_{2}=\left[\begin{array}{c}
Y_{h 2} \\
U_{h 2}
\end{array}\right]
$$

where

$$
\begin{gathered}
Y_{h 1}=\left[\begin{array}{cccc}
y[k] & y[k+1] & \ldots & y[k+j-1] \\
y[k+1] & y[k+2] & \ldots & y[k+j] \\
y[k+2] & y[k+3] & \ldots & y[k+j+1] \\
\vdots & \vdots & & \vdots \\
y[k+i-1] & y[k+i] & \ldots & y[k+j+i-2]
\end{array}\right] \\
Y_{h 2}=\left[\begin{array}{cccc}
y[k+i] & y[k+i+1] & \ldots & y[k+i+j-1] \\
y[k+i+1] & y[k+i+2] & \ldots & y[k+i+j] \\
y[k+i+2] & y[k+i+3] & \ldots & y[k+i+j+1] \\
\vdots & \vdots & & \vdots \\
y[k+2 i-1] & y[k+2 i] & \ldots & y[k+2 i+j-2]
\end{array}\right]
\end{gathered}
$$

and $U_{h 1}, U_{h 2}$ similarly constructed. Both matrix pairs satisfy the $\mathrm{I} / \mathrm{O}$-equation

$$
\begin{aligned}
& Y_{h 1}=\Gamma_{i} \cdot X_{1}+H_{t} \cdot U_{h 1} \\
& Y_{h 2}=\Gamma_{i} \cdot X_{2}+H_{t} \cdot U_{h 2}
\end{aligned}
$$

Theorem 3

If $X_{2}$ is defined as

$$
X_{2}=[x[k+i] \quad x[k+i+1] \quad \ldots \quad x[k+i+j-1]]
$$


then

$$
\operatorname{span}_{\text {row }}\left(X_{2}\right)=\operatorname{span}_{\text {row }}\left(H_{1}\right) \cap \operatorname{span}_{\text {row }}\left(H_{2}\right)
$$

(see (6) for the definition of $H_{1}$ and $H_{2}$ ) so that any basis for this intersection constitutes a valid state vector sequence $X_{2}$ with the basis vectors as the consecutive row vectors.

Note that different choices for a basis differ in a transformation matrix $T$ that transforms a model $A, B, C, D$ into an equivalent model $T^{-1} \cdot A \cdot T, T^{-1} \cdot B, C \cdot T, D$ (Kailath 1980).

Proof

It is first proven that the dimension of the intersection equals $n$. Then, the ( $n$ dimensional) row space of $X_{2}$ is shown to lie within both row spaces.

By making use of the rank property (5), we derive

$$
\operatorname{dim}\left(H_{1}\right)=\operatorname{dim}\left(H_{2}\right)=m i+n
$$

where $\operatorname{dim}(M)$ is a shorthand notation for the dimension of the row space of $M$. This rank property holds equally well for the concatenation of $H_{1}$ and $H_{2}$

$$
H=\left[\begin{array}{l}
H_{1} \\
H_{2}
\end{array}\right]
$$

$\operatorname{dim}\left(H_{1}+H_{2}\right)=\operatorname{dim}(H)=2 m i+n$

Applying Grassmann's dimension theorem

$$
\begin{aligned}
\operatorname{dim}\left(H_{1} \cap H_{2}\right) & =\operatorname{dim}\left(H_{1}\right)+\operatorname{dim}\left(H_{2}\right)-\operatorname{dim}\left(H_{1}+H_{2}\right) \\
& =m i+n+m i+n-2 m i-n
\end{aligned}
$$$$
=n
$$

From (8), we derive

$$
X_{2}=\Gamma_{i}^{+} \cdot Y_{h 2}-\Gamma_{i}^{+} \cdot H_{t} \cdot U_{h 2}=\left[\Gamma_{i}^{+}-\Gamma_{i}^{+} \cdot H_{t}\right]\left[\begin{array}{c}
Y_{h 2} \\
U_{h 2}
\end{array}\right]
$$

where $\Gamma_{i}^{+}$is the pseudo-inverse of $\Gamma_{i}\left(\Gamma_{i}^{+} \cdot \Gamma_{i}=I_{n \times n}\right.$ since $\Gamma_{i}$ has full column rank), which shows that the row space of $X_{2}$ lies within the row space of $H_{2}$. Equally well, $X_{1}$ 's row space lies within $H_{1}$ 's row space. On the other hand, $X_{1}$ and $U_{h 1}$ completely $X_{1}$ 's row space lies with
determine $X_{2}$ through

$$
X_{2}=A^{i} \cdot X_{1}+\left[\begin{array}{llll}
A^{i-1} \cdot B & \ldots & A \cdot B & B
\end{array}\right] U_{h 1}
$$

and since $X_{1}$ 's row space lies within $H_{1}$ 's row space, the same holds true for $X_{2}$ 's row space.

The above theorem allows us to calculate a state vector sequence, making use of measured I/O-data only. Once this state vector sequence is known, the system matrices are easily identified from a set of linear equations, as shown in the next section.

In practice, due to perturbations on the measured data (noise, non-linearity, etc.), it occurs that both row spaces do not intersect. An approximate intersection can be calculated though, using the $n$ first principal vectors (canonical variate analysis), $n$ being determined by (5). As it turns out to be both computationally less demanding and less sensitive to noise on the I/O-data, an alternative procedure is presented. Let the SVD of $H=\left[\begin{array}{l}H_{1} \\ H_{2}\end{array}\right]$ be

$$
H=\left[\begin{array}{ll}
U_{11} & U_{12} \\
U_{21} & U_{22}
\end{array}\right]\left[\begin{array}{cc}
S_{11} & 0 \\
0 & 0
\end{array}\right] V^{t}
$$

where the matrices have the following dimensions

$$
\begin{aligned}
& \operatorname{dim}\left(U_{11}\right)=(m i+l i) \times(2 m i+n) \\
& \operatorname{dim}\left(U_{12}\right)=(m i+l i) \times(2 l i-n) \\
& \operatorname{dim}\left(U_{21}\right)=(m i+l i) \times(2 m i+n) \\
& \operatorname{dim}\left(U_{22}\right)=(m i+l i) \times(2 l i-n) \\
& \operatorname{dim}\left(S_{11}\right)=(2 m i+n) \times(2 m i+n)
\end{aligned}
$$

From

$$
\left[\begin{array}{ll}
U_{12}^{i} & U_{22}^{\prime}
\end{array}\right]\left[\begin{array}{l}
H_{1} \\
H_{2}
\end{array}\right]=0
$$

$$
U_{12}^{t} \cdot H_{1}=-U_{22}^{t} \cdot H_{2}
$$

it follows that the row space of $U_{12}^{t} \cdot H_{1}$ equals the required intersection of the row spaces of $H_{1}$ and $H_{2} \cdot U_{12}^{t} \cdot H_{1}$ contains $2 l i-n$ row vectors, only $n$ of which are linearly independent (dimension of the intersection). Thus, it remains to select $n$ suitable combinations of these row vectors. One straightforward way would consist of taking the SVD of $U_{12}^{\prime} \cdot H_{1}$ in order to compute a basis for its row space. The following theorem gives the outline of a shortcut to this method, replacing the SVD of $U_{12}^{t} \cdot H_{1}-\mathrm{a}[(2 l i-n) \times j]$-matrix where most of the time $j$ is very large-by a $U_{12}^{\prime} \cdot H_{1}-\mathrm{a}$
smaller SVD.

Theorem 4

$$
\begin{aligned}
& \text { Let the SVD of } H=\left[\begin{array}{l}
H_{1} \\
H_{2}
\end{array}\right] \text { be } \\
& \qquad H=\left[\begin{array}{ll}
U_{11} & U_{12} \\
U_{21} & U_{22}
\end{array}\right]\left[\begin{array}{cc}
S_{11} & 0 \\
0 & 0
\end{array}\right] V^{\prime}
\end{aligned}
$$

then the state vector sequence $X_{2}=\left[\begin{array}{lllll}x[k+i] & x[k+i+1] & \ldots & x[k+i+j-1]\end{array}\right]$ can be calculated as

$$
X_{2}=U_{q}^{t} \cdot U_{12}^{t} \cdot H_{1}
$$

where $U_{q}^{t}$-an $[n \times(2 l i-n)]$-matrix accounting for the necessary reduction of 
列 defined through the SVD of $U_{12}^{t} \cdot U_{11} \cdot S_{11}$

$$
U_{12}^{\prime} \cdot U_{11} \cdot S_{11}=\left[\begin{array}{ll}
U_{q} & U_{q}^{1}
\end{array}\right]\left[\begin{array}{cc}
S_{q} & 0 \\
0 & 0
\end{array}\right]\left[\begin{array}{c}
V_{q}^{t} \\
V_{q}^{1 t}
\end{array}\right]
$$

Proof

Since any basis for the row space of $U_{12}^{t} \cdot H_{1}$ is a realization of $X_{2}$ (see above), we first calculate its SVD

$$
\begin{aligned}
& U_{12}^{\prime} \cdot H_{12}=U_{12}^{t}\left[\begin{array}{ll}
U_{11} & U_{12}
\end{array}\right]\left[\begin{array}{cc}
S_{11} & 0 \\
0 & 0
\end{array}\right] V^{\prime} \\
& =\left[U_{12}^{t} \cdot U_{11} \cdot S_{11} \quad 0\right] V^{\prime} \\
& =\left[\begin{array}{ll}
U_{q} \cdot S_{q} \cdot V_{q}^{t} & 0
\end{array}\right] V^{t} \\
& =U_{q}\left[\begin{array}{ll}
S_{q} & 0
\end{array}\right]\left(V \cdot V_{q}\right)^{t}
\end{aligned}
$$

Now, since $U_{q}^{t} \cdot U_{q}=I_{n \times n}$

$$
U_{q}^{t} \cdot U_{12}^{t} \cdot H_{1}=\left[\begin{array}{ll}
S_{q} & 0
\end{array}\right]\left(V \cdot V_{q}\right)^{t}
$$

which is a valid basis for the row space of $U_{12}^{i} \cdot H_{1}$ and thus a realization of $X_{2}$

\section{Identification of system matrice}

Once $X_{2}$ is known, the system matrices can be identified by solving a set of linear equations in a straightforward way

$$
\left[\begin{array}{ccc}
x[k+i+1] & \ldots & x[k+i+j-1] \\
y[k+i] & \ldots & y[k+i+j-2]
\end{array}\right]=\left[\begin{array}{ll}
A & B \\
C & D
\end{array}\right]\left[\begin{array}{lll}
x[k+i] & \ldots & x[k+i+j-2] \\
u[k+i] & \ldots & u[k+i+j-2]
\end{array}\right]
$$

As this (overdetermined) set of equations should be solved in the least-squares sense, the residuals correspond to the noise terms $w[k]$ and $v[k]$ introduced in $\S 2$.

Once again, a computationally more efficient way of computing the system matrices is conceivable, making use of the already calculated SVD of $H$ (concatenation of $H_{1}$ and $H_{2}$ ). The above set of equations may be replaced by a reduced equivalent set, revealing exactly the same least-squares solution.

For compact notation, it is useful to first redefine matrices $H_{1}$ and $H_{2}(6)$ in the following way

$$
H_{1}=\left[\begin{array}{cccc}
u[k] & u[k+1] & \ldots & u[k+j-1] \\
y[k] & y[k+1] & \ldots & y[k+j-1] \\
u[k+1] & u[k+2] & \ldots & u[k+j] \\
y[k+1] & y[k+2] & \ldots & y[k+j] \\
\vdots & \vdots & & \vdots \\
u[k+i-1] & u[k+i] & \ldots & u[k+j+i-2] \\
y[k+i-1] & y[k+i] & \ldots & y[k+j+i-2]
\end{array}\right]
$$

$$
H_{2}=\left[\begin{array}{cccc}
u[k+i] & u[k+i+1] & \ldots & u[k+i+j-1] \\
y[k+i] & y[k+i+1] & \ldots & y[k+i+j-1] \\
u[k+i+1] & u[k+i+2] & \ldots & u[k+i+j] \\
y[k+i+1] & y[k+i+2] & \ldots & y[k+i+j] \\
\vdots & \vdots & & \vdots \\
u[k+2 i-1] & u[k+2 i] & \ldots & u[k+2 i+j-2] \\
y[k+2 i-1] & y[k+2 i] & \ldots & y[k+2 i+j-2]
\end{array}\right]
$$

Notice that Theorem 3 remains valid. We also introduce the following notation $M(p: q, r: s)$ is the submatrix of $M$ at the intersection of rows $p, p+1, \ldots, q$ and columns $r, r+1, \ldots, s ; M(: r: s)$ is the submatrix of $M$ containing columns $r, r+1, \ldots$ $s ; M(p: q,:)$ is the submatrix of $M$ containing rows $p, p+1, \ldots, q$. As an example

$$
H_{1}=H(1: m i+l i,:)
$$

Now let the SVD of $H=\left[\begin{array}{l}H_{1} \\ H_{2}\end{array}\right]$ be

$$
H=U \cdot S \cdot V^{t}
$$

Theorem 5

The system matrices may be identified from the following set of linear equation

$\left[\begin{array}{c}U_{q}^{t} \cdot U_{12}^{\prime} \cdot U(m+l+1:(i+1)(m+l),:) S \\ U(m i+l i+m+1:(m+l)(i+1),:) S\end{array}\right]$

$$
=\left[\begin{array}{ll}
A & B \\
C & D
\end{array}\right]\left[\begin{array}{c}
U_{q}^{t} \cdot U_{12}^{t} \cdot U(1: m i+l i,:) S \\
U(m i+l i+1: m i+l i+m,:) S
\end{array}\right]
$$

(see $\S 3$ for a definition of $U_{q}$ and $U_{12}$ )

$$
\text { Proof }
$$

\section{From $\S 3$ it follows that}

$$
\begin{aligned}
{[x[k+i] \quad \ldots \quad x[k+i+j-1]} & =U_{q}^{t} \cdot U_{12}^{t} \cdot H_{1} \\
& =U_{q}^{t} \cdot U_{12}^{t} \cdot H(1: m i+l i,:) \\
& =U_{q}^{t} \cdot U_{12}^{t} \cdot U(1: m i+l i,:) S \cdot V^{t}
\end{aligned}
$$

Making use of the time-invariance and the block Hankel structure of matrix $H$, we can easily prove that

$$
[x[k+i+1] \quad \ldots \quad x[k+i+j]]
$$

$$
=U_{q}^{\prime} \cdot U_{12}^{t} \cdot H(m+l+1:(i+1)(m+l),:)
$$

$=U_{q}^{t} \cdot U_{12}^{t} \cdot U(m+l+1:(i+1)(m+l),:) S \cdot V^{t}$ 
Also, from the definition of $H$, it follows that

$[u[k+i] \quad \ldots \quad u[k+i+j-1]]=H(m i+l i+1: m i+l i+m,:)$

$$
=U(m i+l i+1: m i+l i+m,:) S \cdot V^{t}
$$

and

$[y[k+i] \quad \ldots \quad y[k+i+j-1]]$

$$
=H(m i+l i+m+1:(m+l)(i+1),:)
$$

$=U(m i+l i+m+1:(m+l)(i+1),:) S \cdot V^{t}$

When (11), (12), (13) and (14) are substituted into the following (overdetermined) set of linear equations

$\left[\begin{array}{ccc}x[k+i+1] & \ldots & x[k+i+j] \\ y[k+i] & \ldots & y[k+i+j-1]\end{array}\right]=\left[\begin{array}{cc}A & B \\ C & D\end{array}\right]\left[\begin{array}{cccc}x[k+i] & \ldots & x[k+i+j-1] \\ u[k+i] & \ldots & u[k+i+j-1]\end{array}\right]$ we obtain

$\left[\begin{array}{c}U_{q}^{t} \cdot U_{12}^{t} \cdot U(m+l+1:(i+1)(m+l),:) S \cdot V^{t} \\ U(m i+l i+m+1:(m+l)(i+1),:) S \cdot V^{t}\end{array}\right]$

$$
=\left[\begin{array}{ll}
A & B \\
C & D
\end{array}\right]\left[\begin{array}{c}
U_{q}^{t} \cdot U_{12}^{t} \cdot U(1: m i+l i,:) S \cdot V^{t} \\
U(m i+l i+1: m i+l i+m,:) S \cdot V^{t}
\end{array}\right]
$$

The common (orthogonal) factor $V^{t}$ can be discarded, thus effectively reducing the number of equations (remember $j \gg \max (m i, l i)$ ), without altering the least-squares solution

$\left[U_{q}^{t} \cdot U_{12}^{t} \cdot U(m+l+1:(i+1)(m+l),:) S\right]$

$U(m i+l i+m+1:(m+l)(i+1),:) S]$

$$
=\left[\begin{array}{ll}
A & B \\
C & D
\end{array}\right]\left[\begin{array}{c}
U_{q}^{t} \cdot U_{12}^{t} \cdot U(1: m i+l i,:) S \\
U(m i+l i+1: m i+l i+m,:) S
\end{array}\right]
$$

Note: The common factor $S$ imposes weights on the different equations. Discarding it would alter the least-squares solution.

\section{Off-line algorithm}

The results of the previous sections are summarized into the following off-line algorithm.

\section{Algorithm 1}

Let $H$ be the concatenation of $H_{1}, H_{2}$, defined by (9) and (10). The system matrices are then obtained as follows.

Step 1. Calculate $U$ and $S$ in the SVD of $H$

$$
H=U \cdot S \cdot V^{t}=\left[\begin{array}{ll}
U_{11} & U_{12} \\
U_{21} & U_{22}
\end{array}\right]\left[\begin{array}{cc}
S_{11} & 0 \\
0 & 0
\end{array}\right] V^{t}
$$
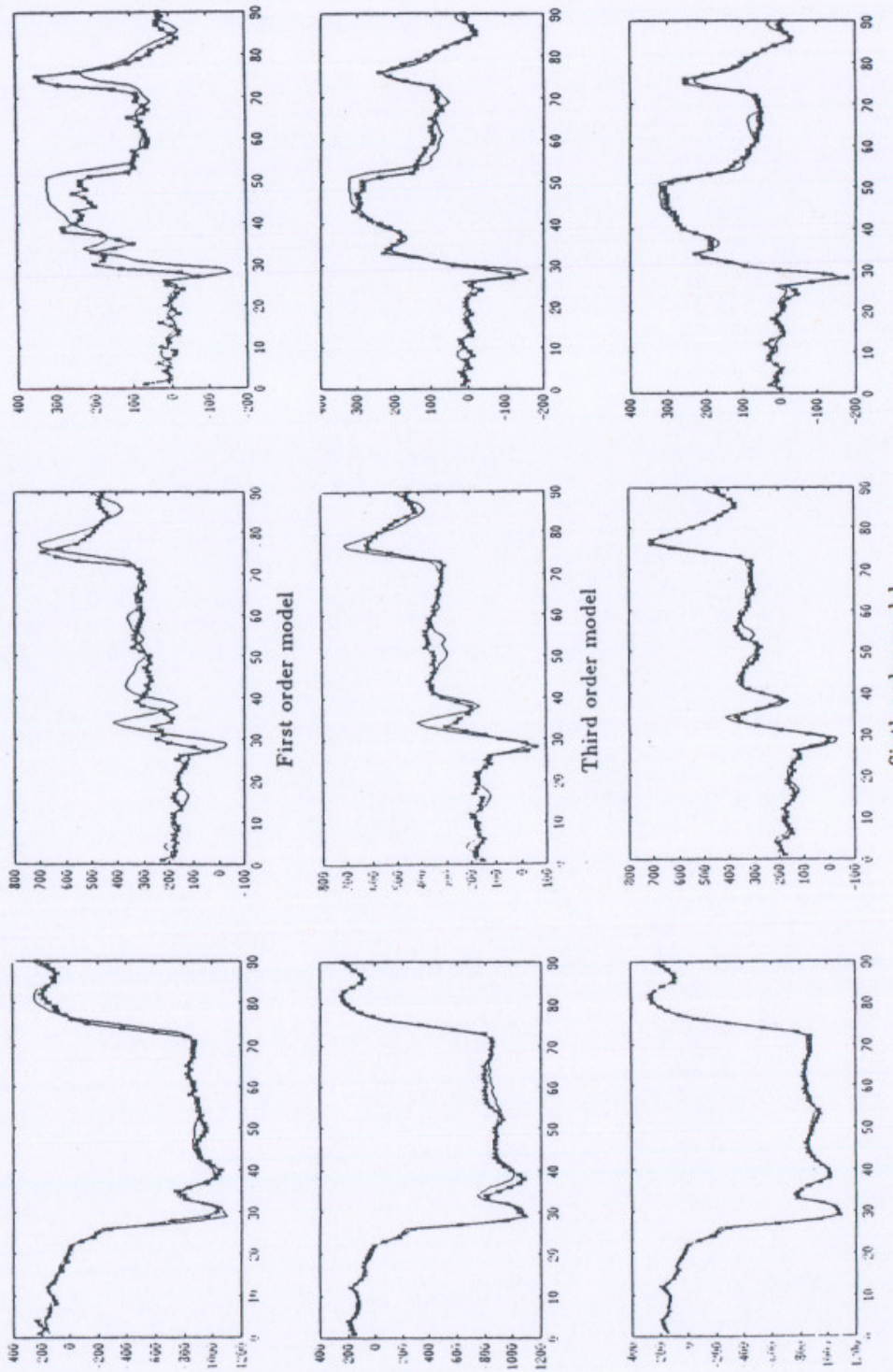
Step 2. Calculate the SVD of $U_{12}^{t} \cdot U_{11} \cdot S_{11}$

$$
U_{12}^{t} \cdot U_{11} \cdot S_{11}=\left[\begin{array}{ll}
U_{q} & U_{q}^{\perp}
\end{array}\right]\left[\begin{array}{cc}
S_{q} & 0 \\
0 & 0
\end{array}\right]\left[\begin{array}{c}
V_{q}^{t} \\
V_{q}^{\perp t}
\end{array}\right]
$$

Step 3. Solve the following set of linear equations

$$
\begin{aligned}
& {\left[\begin{array}{c}
U_{q}^{t} \cdot U_{12}^{t} \cdot U(m+l+1:(i+1)(m+l),:) S \\
U(m i+l i+m+1:(m+l)(i+1),:) S
\end{array}\right]} \\
& =\left[\begin{array}{ll}
A & B \\
C & D
\end{array}\right]\left[\begin{array}{c}
U_{q}^{t} \cdot U_{12}^{t} \cdot U(1: m i+l i,:) S \\
U(m i+l i+1: m i+l i+m,:) S
\end{array}\right]
\end{aligned}
$$

It is worth noticing that the system matrices are ultimately identified from $U$ and $S$ only $\left(H=U \cdot S \cdot V^{i}\right)$, and that the much larger and much more noise-sensitive matrix $V$ is fortunately never used. Even the state vector sequence $X_{2}$ does not need to be constructed explicitly. This reduction turns out to be very useful when an adaptive identification algorithm is constructed (see $\S 6$ ).

\section{Example}

The performance of the algorithm has been evaluated on both simulated and industrial data sets. The following example is due to Prof. R. Guidorzi (University of Bologna) (Guidorzi and Rossi 1974). The I/O-sequence has been obtained under normal operating conditions of a $120 \mathrm{MW}$ power plant (Pont sur Sambre, France), a system with five inputs and three outputs. The identified models (for different system order estimates) have been evaluated by comparing original and simulated outputs, using the original input signals and the identified model (Fig. 1). These simulations demonstrate the remarkable robustness of the identification scheme with respect to over- and underestimation of the system order.

\section{On-line algorithm}

Algorithm 1 is easily converted into an adaptive one, where model updating should account for time-variance. Every time-step a new input-output measurement becomes available, defining a new column to be added to the matrix $H$. On the other hand, older measurements should be discarded by successively deleting columns from $H$. The off-line algorithm of the previous section is then applied to the updated $H$ matrix.

Instead of using this moving window technique, we can also apply exponential weighting. New columns are still added to $H$, but instead of deleting columns, all columns are multiplied by a weighting factor $\alpha(\alpha \leqslant 1)$. This way, a column that was added $q$ time-steps earlier, is weighted with a factor $\alpha^{q}$, thus effectively reducing the contribution of the older data.

Since only $U$ and $S$ in the SVD of $H$ are needed (see $\S 5$ ), $H$ does not need to be constructed explicitly, since the weighting can also be applied to $S$.

Algorithm 2

Initialize $U_{0}=I_{(2 m i+2 l i) \times(2 m i+2 l i)}, S_{0}=0_{(2 m i+2 l i) \times(2 m i+2 l i),} m$ and $l$ being the number of inputs and outputs, respectively, $2 i$ being the number of block rows in the fictitious matrix $H$ for $k=1, \ldots$

Step 1. Construct new column column to be added to $H$, using the $2 i$ latest $\mathrm{I} / \mathrm{O}$ measurements

Step 2. Calculate SVD

$$
U_{k} \cdot S_{k} \cdot V_{k}^{\prime}=\left[\alpha \cdot U_{k-1} \cdot S_{k-1} \quad \text { column }\right]
$$

and partition

$$
U_{k} \cdot S_{k}=\left[\begin{array}{ll}
U_{11} & U_{12} \\
U_{21} & U_{22}
\end{array}\right]\left[\begin{array}{cc}
S_{11} & 0 \\
0 & 0
\end{array}\right]
$$

Step 3. Calculate the SVD of $U_{12}^{\prime} \cdot U_{11} \cdot S_{11}$

$$
U_{12}^{t} \cdot U_{11} \cdot S_{11}=\left[\begin{array}{ll}
U_{q} & U_{q}^{\perp}
\end{array}\right]\left[\begin{array}{cc}
S_{q} & 0 \\
0 & 0
\end{array}\right]\left[\begin{array}{c}
V_{q}^{t} \\
V_{q}^{\perp t}
\end{array}\right]
$$

Step 4. Solve the following set of linear equations

$$
\begin{aligned}
& {\left[\begin{array}{c}
U_{q}^{t} \cdot U_{12}^{\prime} \cdot U(m+l+1:(i+1)(m+l),:) S \\
U(m i+l i+m+1:(m+l)(i+1),:) S
\end{array}\right]} \\
& \quad=\left[\begin{array}{ll}
A & B \\
C & D
\end{array}\right]\left[\begin{array}{c}
U_{q}^{t} \cdot U_{12}^{i} \cdot U(1: m i+l i,:) S \\
U(m i+l i+1: m i+l i+m,:) S
\end{array}\right]
\end{aligned}
$$

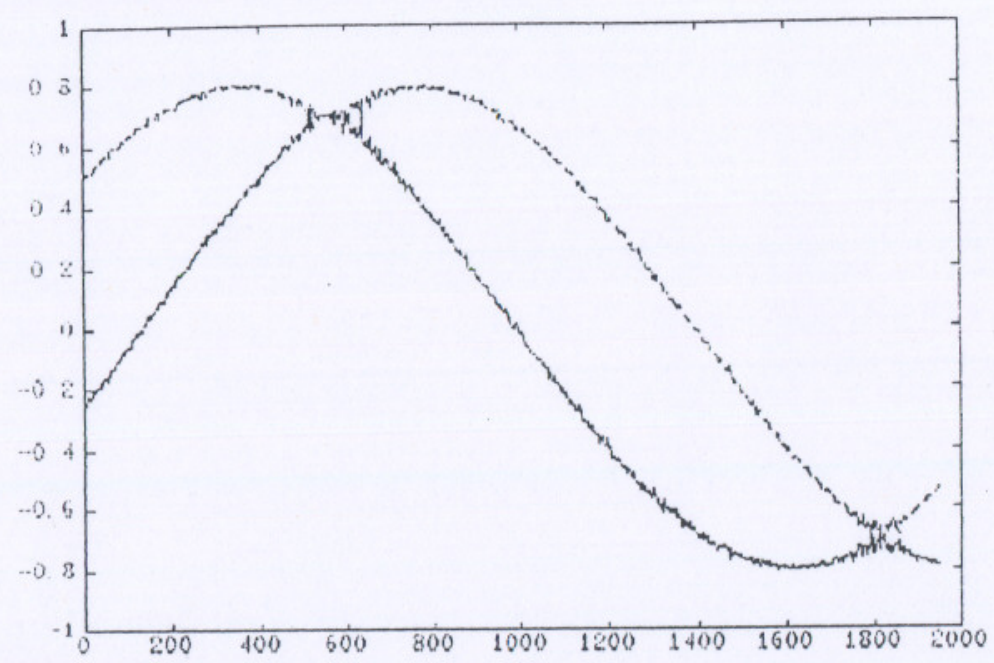

Figure 2. Identified poles for a second-order time-varying system with sinusoidally varying system poles. 
Example

As an example, a second-order time-variant system with two inputs and two outputs and sinusoidally varying system poles is identified. Figure 2 shows the identified system poles when the weighting factor is set equal to $1-2^{-4}$

\section{Conclusion}

A novel strategy for state-space identification from (noisy) $\mathrm{I} / \mathrm{O}$-measurements is presented. The system matrices are identified by only applying numerically stable SVD techniques to a block Hankel matrix (number of columns $\gg$ number of rows), constructed with $\mathrm{I} / \mathrm{O}$-data. As it turns out that only the left singular basis is required, both the computational load and the noise sensitivity are considerably reduced. Moreover, the algorithm is easily converted into an adaptive version for slowly timevarying systems, making use of adaptive SVD algorithms. Extensive simulations have demonstrated the remarkable robustness of the identification scheme with respect to noise and over- and underestimation of the system order.

ACKNOWLEDGMENT

Marc Moonen and Lieven Vandenberghe were supported by the N.F.W.O. (Belgian National Fund for Scientific Research).

\section{REFERENCES}

AKAIKE, H., 1974, I.E.E.E. Trans. autom. Control, 19, 667; 1975, SIAM J. Control, 13, 162. BaraM. Y., 1981, I.E.E.E. Trans. autom. Control, 26, 1225 .

De Moor. B., Moonen, M., VandenBerghe, L., and VANDEWAlLE, J., 1988, Identification of linear state space models with singular value decomposition using canonical correlation analysis. Singular Value Decomposition and Signal Processing, edited by E. Deprettere (Amsterdam: North-Holland), pp. 161-169.

GoluB, G. H., and VAN LOAN, C. F., 1983, Matrix Computations (Baltimore, MD: North Oxford Academic Publishing Co., Johns Hopkins University Press).

GO, B. L. and KALMAN, R., E 1966 . Regelungstechnik, 14, 545.

HotelLiNG, H.. 1936, Biometrika, 28, 321 .

Kallath, T., 1980, Linear System Theory (Englewood Cliffs, NJ: Prentice Hall).

KaLman, R. E.. 1982, System Identification from Noisy Data. Dynamical Systems II, edited by A. R. Bednarek and L. Cesari (New York: Academic Press).

KUNG, S. Y., 1978, A new identification and model reduction algorithm via singular value decomposition. Proc. 12th Asilomar Conf. on Circuits, Systems and Computers, Pacific

Grove. pp. 705-714.
LARIMORE, W. E., 1984, System identification, reduced-order filtering and modelling via canonical variate analysis. Proc. 1983 Automatic Control Conf., San Francisco.

RAMOS. J. A., and VERRIEST, E. I., 1984, A unifying tool for comparing stochastic realization algorithms and model reduction techniques. Proc. 1984 Automatic Control Conf., San ZEIGER, H. P., 